\title{
Dor pós-operatória em Endodontia: revisão de literatura
}

Post-operative pain in Endodontics: literature review

Dolor post-operatoria en Endodoncia: revisión de literatura

Tamires Vieira de SOUSA 1

José Henrique de Araújo $\mathbf{C R U Z}^{2}$

Kamilla Alencar SOUSA ${ }^{\mathbf{1}}$

Tássia Cristina de Almeida Pinto SARMENTO3

Rosana Araújo ROSENDO ${ }^{4}$

${ }^{1}$ Cirurgiã-Dentista pelo Curso de Graduação em Odontologia. Centro de Saúde e Tecnologia Rural, Universidade Federal de Campina Grande, UFCG 58708-110 Patos - PB, Brasil

${ }^{2}$ Cirurgião-Dentista pelo Curso de Graduação em Odontologia. Centro de Saúde e Tecnologia Rural, Universidade Federal de Campina Grande, UFCG 58708-110 Patos - PB, Brasil, e Pós-Graduando em Ortodontia e Ortopedia Funcional dos Maxilares pelas Faculdades Unidas do Norte de Minas, FUNORTE, Campina Grande - PB, Brasil

${ }^{3}$ Professora Doutora do Curso de Graduação em Odontologia. Centro de Saúde e Tecnologia Rural, Universidade Federal de Campina Grande, UFCG 58708-110 Patos - PB, Brasil

${ }^{4}$ Professora Doutora do Curso de Graduação em Odontologia. Centro de Saúde e Tecnologia Rural, Universidade Federal de Campina Grande, UFCG 58708-110 Patos - PB, Brasil

\section{Resumo}

Introdução: A endodontia é um ramo da odontologia baseado na saúde do sistema de canais radiculares, promovendo limpeza destes, frequentemente associada a eventos de dor pós-operatória. Vários fatores têm sido sugeridos como propiciadores deste sintoma. Objetivo: Este trabalho visa reunir dados da literatura que possam explicitar a ocorrência e intensidade da sintomatologia dolorosa pós-operatória em endodontia e quais variáveis podem estar envolvidas neste processo. Material e Método: Utilizou-se de 43 artigos científicos retirados das bases de dados: SciELO, MEDLINE, Lilacs, PubMed, BBO e BVS, encontrados em português, inglês e espanhol, usando os descritores: Dor Pós-Operatória, Endodontia, Polpa Dentária; com restrição de período de 2009 a 2019. Resultados: A dor pós-operatória em endodontia é relatada em aproximadamente metade dos casos, sendo a dor pré-operatória um fator fortemente associado. O número de sessões clínicas não exerceu diferenças na dor, bem como o uso de diferentes técnicas de instrumentação avaliadas. Polpas vitais exercem maior incidência deste sintoma. Ampliação de forame demonstrou-se mais associada à dor, enquanto a execução da patência foraminal mostrou capacidade de não produzi-la. Hidróxido de cálcio, clorexidina e pasta de antibiótico mostraram-se importantes medicações intracanal, bem como hipoclorito de sódio, clorexidina e ácido etilenodiaminotetracético foram soluções irrigadoras satisfatórias. Conclusão: Todas as variáveis evidenciaram alguma relação com a dor pós-operatória em endodontia, no entanto não se exibiram de forma expressiva, sugerindo que mais estudos são necessários sobre este tema.

Descritores: Dor Pós-Operatória; Endodontia; Polpa Dentária.

\section{Abstract}

Introduction: Endodontics is a branch of dentistry based on the health of the root canal system, promoting cleaning of these, often associated with postoperative pain events. Several factors have been suggested as propitiators of the symptom. Objective: This paper aims to gather data from the literature that can explain the occurrence and intensity of postoperative pain symptomatology in endodontics and which variables may be involved in this process. Material and Method: We used 43 scientific articles from the databases: SciELO, MEDLINE, Lilacs, PubMed, BBO and BVS, found in Portuguese, English and Spanish, using the descriptors: Post Operative Pain, Endodontics, Dental Pulp; with period restriction from 2009 to 2019. Results: Post-operative endodontic pain is reported in approximately half of the cases, with preoperative pain being a strongly associated factor. The number of clinical sessions did not exert differences in pain, as well as the use of different instrumentation techniques evaluated. Vital pulps exert a higher incidence of this symptom. Enlargement of foramen was shown to be more associated with pain, while the execution of foraminal patency showed the capacity of not producing it. Calcium hydroxide, chlorhexidine and antibiotic paste showed important intracanal medications, as well as sodium hypochlorite, chlorhexidine and ethylenediaminetetraacetin acid werw satisfactory irrigating solutions. Conclusion: All variables showed some relationship with postoperative pain in endodontics, however they did not express themselves expressively, suggesting that more studies are needed on this topic.

Descriptors: Postoperative Pain; Endodontics; Dental Pulp.

\section{Resumen}

Introdución: La endodoncia es una rama de la odontología basada en la salud del sistema de canales radiculares, promoviendo limpieza de éstos, frecuentemente asociada a eventos de dolor postoperatorio. Varios factores han sido sugeridos como propiciadores de este síntoma. Objetivo: Este trabajo busca reunir datos de la literatura que puedan explicitar la ocurrencia e intensidad de la sintomatología dolorosa postoperatoria en endodoncia y cuáles variables pueden estar involucradas en este proceso. Materiales y Métodos: Se utlizaron 43 artículos científicos extraídos de las bases de datos SciELO, MEDLINE, Lilacs, PubMed, BBO y BVS, se encuentran en portugués, español e inglés, utilizando los descriptores: dolor postoperatorio, endodoncia, pulpa dental; con restricción de período de 2009 a 2019. Resultados: El dolor postoperatorio en endodoncia es reportado en aproximadamente la mitad de los casos, siendo el dolor preoperatorio un factor fuertemente asociado. El número de sesiones clínicas no ejerció diferencias en el dolor, así como el uso de diferentes técnicas de instrumentación evaluadas. Las pulpas vitales ejercen mayor incidencia de este síntoma. La amplificación de foramen se demostró más asociada al dolor, mientras que la ejecución de la patencia fueroninal mostró capacidad de no producirla. El hidróxido de cálcio, la clorexidina y la pasta de antibiótico se mostraron importantes medicamentos intracanales, así como hipoclorito de sodio, clorexidina y acido etilendiaminotetracético, fueron soluciones de riego satisfactorias. Conclusión: Todas las cariables evidenciaron alguna relación con el dolor postoperatorio en endodoncia, sin embargo no se exhibieron de forma expresiva, sugiriendo que más estudios son necesarios sobre este tema.

Descriptores: Dolor Postoperatorio; Endodoncia; Pulpa Dental.

INTRODUÇÃO

O tratamento endodôntico tem por objetivo a eliminação de microrganismos por meio da desinfecção do sistema de canais radiculares, contando com um correto preparo químico mecânico, uso de substâncias coadjuvantes e uma obturação adequada com 
compatibilidade biológica dos cimentos endodônticos. Estas são condições satisfatórias para possibilitar o sucesso na terapia em endodontia ${ }^{1,2}$.

A Associação Internacional para o Estudo da Dor (IASP) caracteriza este evento como sendo uma experiência sensorial desagradável relacionada a noções subjetivas de emoção, memória e cognição que envolve um dano ao nervo periférico, descrita como uma associação entre estímulos sensoriais, centrais e periféricos ${ }^{3}$.

Gotler et al. $^{4}$ postulam a terapia endodôntica como prática odontológica que se apresenta mais relacionada à dor pósoperatória, destacando que é imprescindível para o profissional estar apto a saber lidar com esta sintomatologia desagradável, já que é descrita como uma situação comum aos atendimentos. Orientar ao paciente sobre a possibilidade do surgimento da dor pode fazer com que haja um aumento no limiar de percepção da mesma, bem como explicar acerca desta possibilidade pode fazer com que haja o estabelecimento de uma maior relação de confiança entre profissional e paciente. $O$ cirurgião-dentista deve estar atento para os cuidados com a dor pós-operatória logo após seu aparecimento inicial, evitando que a resposta intensifique.

A dor pós-operatória (DPO) é uma consequência possível de ocorrer durante 0 tratamento endodôntico, visto que o mesmo não é capaz de eliminar todo o processo inflamatório existente de maneira imediata. Alguns fatores são sugeridos como propiciadores da inflamação aos tecidos perirradiculares, relacionados com o surgimento da dor pósoperatória, dentre eles: agressões químicas, mecânicas, microbiológicas e/ou a associação destas $^{5,6}$.

Diante do exposto, este trabalho objetiva estudar a dor pós-operatória em endodontia por meio de revisão de literatura acerca do tema, analisando artigos que discorram sobre a frequência e ocorrência desta sintomatologia, bem como compreendendo as causas e variáveis que possam estar envolvidas na dor relacionada ao tratamento endodôntico.

\section{MATERIAL E MÉTODO}

Trata-se de uma revisão de literatura narrativa, buscando estudos que discorrem sobre dor pós-operatória em endodontia. Como embasamento para a pesquisa foi utilizados artigos científicos retirados das seguintes bases de dados: SciELO (Scientific Eletronic Library Online), MEDLINE (Medical Literature Analysis and Retrieval Sistem Online), Lilacs (Centro
Latino-Americano e do Caribe de Informação em Ciências da Saúde), PubMed (U.S. National Library of Medicine), BBO (Bibliografia Brasileira de Odontologia), BVS (Biblioteca Virtual em Saúde). Para a pesquisa dos artigos, foram utilizados como descritores isolados ou combinados: Dor Pós-Operatória, Endodontia, Polpa Dentária; com restrição de período de 2009 a 2019. No total, 43 artigos constituíram o estudo, encontrados em três idiomas: português, inglês e espanhol. Como critério de inclusão, foram selecionados apenas artigos que abordavam os conceitos de dor pósoperatória e endodontia de maneira específica. Para critério de exclusão, foram descartados trabalhos que não continham o estudo na íntegra, como resumos. Com o intuito de promover uma melhor compreensão, a discussão foi dividida em tópicos de acordo com as variáveis estudadas.

RESULTADOS E DISCUSSÃO

- Frequência e intensidade da dor

Segura-Egea et al. ${ }^{7}$ avaliaram a dor pósoperatória em tratamento de canal radicular, levando em consideração variáveis como tipo de dente, idade, sexo, estado periapical, diagnóstico pulpar e tempo de sessão clínica. $O$ método para avaliação da sintomatologia foi a escala analógica visual, com escores de zero a dez, onde a média de respostas obtidas foi em torno de 1,2 e $0,8 \%$ de intensidade da dor. Pouco mais da metade dos pacientes relatou ausência deste sintoma. No entanto, observouse que pacientes mais jovens, tratamento em dentes posteriores e com tempo clínico de sessão superior a 45 minutos demonstraram uma incidência maior de dor. ${ }^{7}$

Pak e White $^{8}$ realizaram revisão sistemática denotando que a grande maioria dos casos de dor estava relacionada ao prétratamento endodôntico, uma pequena parcela associada à dor após 24 horas de atendimento e em uma semana de pós-operatório, essa sintomatologia diminuiu significativamente. A intensidade foi classificada como moderada, principalmente alusiva à dor pré-operatória. De mesmo modo, Rosso et al. ${ }^{9}$ afirmam em seu estudo, que a dor pós-operatória esteve presente com maior incidência em casos de sintomatologia prévia.

No trabalho desenvolvido por Waskievicz et al. ${ }^{10}$, com um total de 302 prontuários de pacientes com tratamento endodôntico realizado, notou-se que o índice de ausência de dor foi maior, em torno de $2 / 3$ dos casos. Do $1 / 3$ restante que referiu DPO, a maior parte classificou como tolerável sobre a intensidade 
da sintomatologia. Alí et al. ${ }^{11}$, em estudo clínico, observaram que a dor pré-operatória é a variável que mais fortemente está associada ao surgimento da dor após o tratamento. Nesse estudo foram tratados endodonticamente 270 elementos dentários verificando, através de questionário e escala visual analógica com escores de zero a dez, em que a intensidade da sintomatologia registrada na escala foi entre dois e três. Oliveira e Rocha ${ }^{12}$ corroboram os achados de Alí et al. ${ }^{11}$, os quais destacaram em seu trabalho que existe uma relação de incidência de casos de DPO em endodontia, quando associados à dor pré-operatória.

\section{- Número de sessões clínicas}

Su et al. $^{13}$, em seu trabalho de revisão de literatura, pontuam que a DPO foi significativamente menor nos casos de endodontia em sessão única, principalmente nas primeiras horas após a terapia endodôntica. Já Rosso et al. ${ }^{9}$ realizaram revisão sistemática que diferiu dos resultados encontrados por Su et al. ${ }^{13}$, concluindo que múltiplas sessões mostraram menor incidência de dor pósoperatória, sugerindo que este fato pode ser explicado pelo uso do hidróxido de cálcio entre as sessões.

Singh e $\mathrm{Garg}^{14}$ analisaram 188 pacientes, metade tratada endodonticamente em sessão única e o restante em sessão múltipla, concluindo assim, não existir diferença considerável entre as duas modalidades propostas. Endo et al. ${ }^{15}$ corroboram os resultados supracitados, após realizarem uma síntese acerca da influência da dor pósoperatória em endodontia, defendendo que a sessão única oferecia maiores riscos de DPO.

Para Rosa ${ }^{16}$, diante dos dados obtidos em sua revisão literária, a terapia endodôntica em sessão única não exerce influência no aumento ou redução da sintomatologia pósoperatória. Já Manfredi et al. ${ }^{17}$ encontraram resultados diferentes, ao afirmarem que a endodontia em sessão única demonstrou maiores índices de DPO em até uma semana após o procedimento. O êxito do tratamento endodôntico está relacionado a um melhor conhecimento e domínio de aspectos anatômicos e técnicos, bem como de um maior combate à infecção, na remissão da sintomatologia dolorosa, não estando tão envolvido o número de sessões clínicas necessárias ${ }^{18}$.

\section{- Diagnóstico pulpar}

Segura-Egea et al. $^{7}$ afirmam que um aumento na percepção da sintomatologia foi observado em dentes com pulpite irreversível e periodontite apical aguda, se comparado a polpas necróticas e periodontite apical crônica. Divergindo deste estudo, El Mubarak et al. ${ }^{19}$ observaram que $13,7 \%$ casos de sintomatologia intensa estiveram relacionados com polpa não vital, enquanto $7,8 \%$ casos desta dor foram referidos em polpas vitais, mostrando assim, uma maior associação de dor pós-operatória em polpas sem vitalidade.

Farzana et al. $^{20}$ demonstraram através de pesquisa clínica, que não houve distinções consideráveis quanto à dor em dentes com e sem vitalidade pulpar. Nos casos em que a sintomatologia estava presente, esta foi manifestada como de intensidade leve e moderada. Este trabalho discorda dos achados de Gotler et al. ${ }^{3}$ que constataram que a presença e incidência da dor pós-operatória foi expressivamente maior nos casos em que a polpa respondeu positivamente aos testes de sensibilidade, sendo principalmente relatada nas primeiras seis horas após sessão.

$\mathrm{Na}$ pesquisa clínica de Waskievicz et al. ${ }^{10}$ observou-se que dos 93 casos onde houve dor pós-operatória, uma parte significativa estava relacionada a polpas vitais, associadas também a uma maior intensidade da sintomatologia. A Tabela 1 reúne propostas sobre o número de sessões e diagnóstico pulpar.

Tabela 1: Relação: número de sessões e diagnóstico pulpar acerca da dor.

\begin{tabular}{|c|c|c|}
\hline Artigo & Tipo de estudo & $\mathrm{N}^{0}$ de sessões clínicas \\
\hline $\begin{array}{c}\text { El Mubarak et al. }{ }^{19} \\
(2010)\end{array}$ & Pesquisa clínica & $\begin{array}{l}\text { Sem diferença acerca do } \mathrm{n}^{0} \\
\text { de sessões na dor }\end{array}$ \\
\hline Su et al. 13 (2011) & Revisão de literatura & Menos dor em sessão única \\
\hline $\begin{array}{l}\text { Singh e Garg' }{ }^{14} \\
\text { (2012) }\end{array}$ & Pesquisa clínica & Sem diferença considerável \\
\hline Artigo & Tipo de estudo & Diagnóstico pulpar \\
\hline $\begin{array}{c}\text { Segura-Egea et al. } 7 \\
(2009)\end{array}$ & Pesquisa clínica & Mais dor em polpas vitais \\
\hline Gotler et al. ${ }^{3}$ (2012) & Pesquisa clínica & $\begin{array}{l}\text { Mais dor expressivamente } \\
\text { em polpas vitais }\end{array}$ \\
\hline Alí et al..1 (2015) & Pesquisa clínica & Sem diferença considerável \\
\hline
\end{tabular}

- Instrumentação

Gurgel-Filho et al. ${ }^{21}$ verificaram o efeito do alargamento foraminal na incidência da sintomatologia dolorosa, ao realizarem pesquisa clínica com 40 dentes, em que o grupo 1 recebeu ampliação foraminal até a lima \#30, enquanto o grupo 2 não recebeu ampliação, ambos com comprimento de trabalho de 1 a 2 $\mathrm{mm}$ do ápice, comprovado mediante uso de localizador foraminal. Evidenciou-se que em ambos, a incidência de dor foi mínima, não havendo distinções relevantes entre os grupos. Pasqualini et al. ${ }^{6}$, em um estudo clínico com o intuito de averiguar a relação da dor pósoperatória relacionado à glide path, utilizando o sistema rotatório Path File (PF) de níquel titânio (Ni-Ti) em um grupo e instrumentação manual com limas tipo $\mathrm{K}$ de aço inoxidável em outro, perceberam que o sistema rotatório $\mathrm{PF}$ de $\mathrm{Ni}$ - $\mathrm{Ti}$ 
proporcionou menor ocorrência de dor, se comparado aos instrumentos manuais.

Kirchhoff et al. ${ }^{22}$ analisaram a quantidade de detritos extruídos no preparo de canais radiculares de 72 dentes, distribuídos para utilização de quatro diferentes técnicas de instrumentação: 1) Sistema Rotatório ProTaper Next; 2) Reciprocante WaveOne; 3) Giratório / recíproco Twisted File Adaptive (TFA); 4) SelfAdjusting File (SAF). Os detritos extruídos foram coletados e pesados para avaliação através de testes. Todas as técnicas empregadas desencadearam alguma quantidade de extrusão de detritos, porém o grupo que o sistema SAF atuou, apresentou uma porção maior de resíduos. Neelakantan e Sharma ${ }^{23}$ (2015) em estudo clínico avaliando a influência de sistema rotatório One Shape e sistema Reciprocante VDW em 605 pacientes, constataram que o sistema reciprocante esteve relacionado a uma menor incidência de sintomatologia pósoperatória.

Cruz-Júnior et al. ${ }^{24}$ encontraram resultados divergentes de Gurgel-Filho et al. ${ }^{21}$, quando realizaram um estudo clínico com 46 pacientes, divididos em grupo que receberia ampliação foraminal, com comprimento de trabalho (CT) a 0,0 $\mathrm{mm}$ do ápice e grupo sem ampliação, com CT a $1 \mathrm{~mm}$ do ápice. Concluiuse que ambos tiveram reduzida incidência de dor pós-operatória, relatada de intensidade leve, mais observada nas primeiras 24 horas, apresentando-se com mais ocorrência no grupo dos pacientes que receberam a ampliação no forame.

Saini et al. $^{25}$ corroboram com CruzJúnior et al. $^{24}$ ao examinarem clinicamente o impacto que a ampliação de forame teria sobre a presença de dor, podendo concluir que a incidência maior de DPO associou-se ao grupo onde 0 alargamento foraminal foi realizado.

Çiçek et al. ${ }^{26}$ desenvolveram uma pesquisa clínica utilizando três técnicas de instrumentação para avaliação da dor, em que os pacientes foram divididos para serem tratados igualmente em três grupos pelas técnicas: 1) Step-back modificada, utilizando instrumentos manuais de aço inoxidável; 2) Instrumentos reciprocantes (WaveOne); 3) Instrumentos rotatórios (ProTaper Next). O grupo de pacientes tratados através da técnica step-back modificada com instrumentos manuais de aço inoxidável demonstrou uma incidência expressivamente menor de DPO.

Uma meta-análise realizada por Hou et al. ${ }^{27}$, avaliando a sintomatologia dolorosa após terapia endodôntica utilizando sistemas rotatórios e reciprocantes, evidenciou que a incidência de dor esteve mais relacionada nos casos onde foram usados instrumentos reciprocantes. Este estudo demonstrou variações ao trabalho de Çiçek et al. ${ }^{26}$, no qual não houve diferença entre o uso destes dois sistemas no aparecimento da dor. Souza et al. $^{28}$ observaram que o sistema reciprocante mostrou menos dor intensa após 48 horas do tratamento endodôntico quando comparado com o rotatório. Para os autores, o movimento rotatório respondeu negativamente à dor pósoperatória do tratamento endodôntico.

\section{- Patência e comprimento de trabalho}

Ribeiro et al. ${ }^{29}$ afirmaram que a patência foraminal não expõe diferenças significativas na quantidade de detritos extruídos e não efetua uma limpeza maior, realizando uma pesquisa laboratorial com 30 elementos dentários extraídos, inundados com tinta nanquim e divididos em dois grupos, onde o primeiro seria submetido à patência foraminal com uso de lima endodôntica tipo $\mathrm{K} \# 15$ e o segundo sem o procedimento de limpeza. Para avaliação, os debris foram coletados e analisados em microscópio estereoscópico.

Tuncer e Gerek $^{30}$ verificaram a influência da determinação do comprimento de trabalho com uso de localizador apical eletrônico, através de uma pesquisa clínica em 220 pacientes, os quais foram separados em dois grupos, o primeiro com determinação do CT por meio de localizador apical eletrônico Root ZX, o segundo teve seu comprimento de trabalho determinado por odontometria visual, por meio de radiografias. Em ambos foi usado a lima tipo $\mathrm{K}$ número \#15. A dor foi avaliada em um intervalo de tempo de quatro a 48 horas após procedimento, sendo mais relatada nas primeiras quatro a seis horas. Concluiu-se que a dor pós-operatória não demonstrou distinções na incidência da sintomatologia com comprimento de trabalho determinado por localizador foraminal ou radiografia.

Arora et al. $^{31}$ analisaram 68 pacientes, divididos em dois grupos, no qual para a porção de pacientes que receberia a patência utilizouse uma lima endodôntica tipo $\mathrm{K} \# 10$, e o grupo controle não recebeu a patência. Os pacientes foram analisados em até sete dias após tratamento, resultando em presença de DPO em $43 \%$ no geral dos casos, sendo esta menor nos pacientes que receberam a patência foraminal, porém a diferença não foi expressiva entre os grupos. Pauletto e Bello ${ }^{32}$ apontam que 0 procedimento de patência foraminal assegura um maior êxito clínico, pelo fato de significar a limpeza de toda a extensão dos canais radiculares, resultando em índice reduzido de 
dor pós-operatória, fator importante para determinação do sucesso.

- Soluções irrigadoras e medicações

Asgary e $\mathrm{Eghbal}^{33}$ analisaram a influência de um cimento endodôntico rico em cálcio no surgimento de sintomatologia dolorosa pós-operatória em endodontia. O grupo que recebeu o curativo à base de cálcio demonstrou um alívio da dor em menos horas do que o grupo sem medicação, sendo 18 e 36 horas para a remissão do sintoma, respectivamente, indicando que a medicação intracanal à base de cálcio contribui para redução da dor.

Bashetty e Hedge ${ }^{34}$ analisaram o efeito de duas soluções irrigadoras (hipoclorito de sódio a 5,25\% e clorexidina em gel à $2 \%$ ) em 40 dentes avaliados no intervalo de tempo de seis horas até sete dias de pós-operatório e concluíram que a incidência maior de DPO esteve associada ao grupo que recebeu 0 hipoclorito de sódio como substância auxiliar, sendo esta sintomatologia mais relatada em seis horas após o procedimento. Este estudo discorda de Almeida et al. $^{35}$, que evidenciam após ensaio clínico realizado com 126 pacientes, divididos em dois grupos, um usando clorexidina em gel à $2 \%$ e outro utilizando hipoclorito de sódio à 5,25\% como solução irrigadora, analisados no período de tempo de 24 horas a sete dias após a sessão, que não houve distinções consideráveis entre os grupos em relação à incidência de dor, sendo esta mínima, mais observada em até 24 horas após terapia.

Singh et al. ${ }^{36}$ elaboraram um estudo clínico para testar a influência de diferentes medicações intracanais na incidência da dor pós-operatória. A amostra constituiu-se de 64 dentes tratados endodonticamente, os quais foram distribuídos em quatro grupos que receberam como medicação intracanal: 1) pasta de hidróxido de cálcio + clorexidina em gel $2 \%$; 2) clorexidina em gel $2 \%$; 3) pasta de hidróxido de cálcio; 4) placebo: sem medicação. Após avaliação da dor, verificou-se que uma menor incidência desta sintomatologia esteve relacionada aos grupos que foram medicados com pasta de hidróxido de cálcio + clorexidina e quando a clorexidina foi usada isolada, grupos 1 e 2 respectivamente. Este trabalho corrobora com Anjaneyulu e Nivedhitha ${ }^{37}$ que afirmam que o hidróxido de cálcio sozinho não é tão eficaz na redução da incidência de DPO, completando que este torna-se mais satisfatório quando associado à clorexidina em gel a $2 \%$ ou 0 monoclorofenol canforado.

Vieyra et al. ${ }^{38}$ desenvolveram um estudo com 216 pacientes com indicação de tratamento endodôntico, em que todos receberam $5 \mathrm{~mL}$ de ácido etilenodiaminotetracético (EDTA) e $10 \mathrm{~mL}$ de solução salina como solução irrigadora, diferindo apenas acerca da temperatura em que estes foram aplicados nos canais radiculares. Evidenciou-se que o EDTA atua como uma importante solução irrigadora, e que a incidência de DPO foi menor nos casos em que este foi usado resfriado, em temperaturas de 2,5 e 4 graus Celsius, se comparado a esta solução utilizada em temperatura ambiente, apesar da diferença entre os grupos não ter sido significativa.

\section{A Tabela 2 apresenta propostas} relacionadas à técnica de instrumentação, patência foraminal, irrigação e medicação.

Tabela 2. Técnicas de instrumentação, patência foraminal, irrigação e medicação e sua relação com a dor

\begin{tabular}{|c|c|c|}
\hline Artigo & Tipo de estudo & Técnica de instrumentação \\
\hline $\begin{array}{c}\text { Gambarini et } \\
\text { al.39(2013) }\end{array}$ & Pesquisa clínica & Mais dor em reciprocante Wave One \\
\hline $\begin{array}{l}\text { Kherlakian et al. } 4^{\circ 0} \\
\text { (2016) }\end{array}$ & Pesquisa clínica & $\begin{array}{c}\text { Sem diferença considerável entre } \\
\text { rotatório e reciprocantes }\end{array}$ \\
\hline Relvas et al.41 (2016) & Pesquisa clínica & $\begin{array}{c}\text { Sem diferença considerável entre } \\
\text { Protaper Next (17,9\% dor) e Reciproc } \\
(15,3 \%) \\
\end{array}$ \\
\hline Artigo & Tipo de estudo & Patência foraminal \\
\hline $\begin{array}{c}\text { Ensinas et al. }{ }^{42} \\
(2015)\end{array}$ & Pesquisa clínica & $\begin{array}{l}\text { Sem diferença entre o grupo que } \\
\text { recebeu patência e o que não recebeu }\end{array}$ \\
\hline Arora et al..$^{31}(2016)$ & Pesquisa clínica & $\begin{array}{l}\text { Menor dor em pacientes que } \\
\text { receberam a patência }\end{array}$ \\
\hline $\begin{array}{l}\text { Pauletto e Bello }{ }^{32} \\
(2018)\end{array}$ & $\begin{array}{l}\text { Revisão de } \\
\text { literatura }\end{array}$ & $\begin{array}{l}\text { Menor dor quando realizado patência } \\
\text { foraminal }\end{array}$ \\
\hline Artigo & Tipo de estudo & Irrigação e medicação \\
\hline $\begin{array}{l}\text { Asgary e Eghbal } 33 \\
\text { (2010) }\end{array}$ & Pesquisa clínica & $\begin{array}{l}\text { Medicação à base de cálcio } \\
\text { contribuiu para remissão da dor }\end{array}$ \\
\hline $\begin{array}{c}\text { Anjaneyulu e } \\
\text { Niveditha }^{37}(2014) \\
\end{array}$ & $\begin{array}{c}\text { Revisão } \\
\text { sistemática }\end{array}$ & $\begin{array}{l}\text { Hidróxido de cálcio atua melhor } \\
\text { combinado à outra substância }\end{array}$ \\
\hline $\begin{array}{c}\text { Bourreau, Soares e } \\
\text { Souza-Filho43 (2015) }\end{array}$ & Pesquisa clínica & $\begin{array}{c}\text { Hipoclorito de sódio } 5,25 \% \text { e } \\
\text { clorexidina em gel } 2 \% \text { apresentaram } \\
\text { resultados similares à dor }\end{array}$ \\
\hline Silva et al.44 (2015) & Pesquisa clínica & $\begin{array}{l}\text { Hipoclorito de sódio } 5,25 \% \text { e } \\
\text { clorexidina em gel } 2 \% \text { são similares e } \\
\text { bons irrigantes em endodontia }\end{array}$ \\
\hline
\end{tabular}

CONCLUSÃO

A dor pós-operatória em endodontia é relativamente habitual, relatada como de intensidade leve ou moderada, demonstrando forte relação com a dor pré-operatória, com mais frequência nas primeiras horas após terapia. O número de sessões clínicas não indica influência significativa acerca da sintomatologia dolorosa, enquanto a incidência da mesma está mais relacionada a polpas vitais. Todas as técnicas de instrumentação avaliadas contribuem para extrusão de detritos e consequentemente dor. A ampliação de forame relaciona-se a uma maior incidência de dor, e patência foraminal demonstra capacidade de não produzi-la. As soluções irrigadoras e medicações intracanal avaliadas são satisfatórias acerca da dor em endodontia. Contudo, sugere-se que mais estudos são necessários acerca deste tema, visto que os resultados não se apresentaram de forma tão significativa.

REFERÊNCIAS

1. Costa GM. Fatores associados ao estado periapical de dentes tratados endodonticamente: um enfoque multidisciplinar 
[dissertação]. (Mestrado). Diamantina: Faculdade de Ciências Básicas, Universidade Federal dos Vales do Jequitinhonha e Mucuri; 2012.

2. Zoletti GO, Carmo FL, Pereira EM, Rosado AS, Siqueira-Júnior JF, Santos KRN. Comparison of endodontic bacterial community structures in root canal treated teeth with or without apical periodontitis. J Med Microbiol. 2010;59 (11):1360-64.

3. Bahia EB. Dor em urgência odontológica: uso de anti-inflamatórios, corticóides e analgésicos em casos de pulpite aguda irreversível [tese]. Bauru: Faculdade de Odontologia de Bauru. Universidade de São Paulo; 2016.

4. Gotler M, Bar-Gil B, Ashkenazi M. Postoperative pain after root canal treatment: a prospective cohort study. Int J Dent. 2012; 2012:310467.

5. Alves VO. Endodontic flare-ups: a prospective study. Oral Surg Oral Med Oral Pathol Oral Radiol Endod. 2010;110(5):e68-e72.

6. Pasqualini D, Mollo L, Scotti N, Cantatore G, Castelucci A, Migliaretti $G$ et al. Postoperative pain after manual and mechanical glide path: $A$ randomized clinical trial. J Endod. 2012;38(1): 32-6.

7. Segura-Egea JJ, Cisneros-Cabello R, LlamasCarreras JM, Velasco-Ortega E. Pain associated with root canal treatment. Int Endod J 2009;42(7):614-20.

8. Pak JG, White SN. Pain prevalence and severity before, during, and after root canal treatment: A systematic review. J Endod. 2011; 37(4):429-38.

9. Rosso BC, Pereira KFS, Boretti VH, Arashiro FN, Guerisoli DMZ, Yoshinari GH. Dor pósoperatória em dentes com Infecções após única ou múltiplas sessões-Revisão sistemática. Pesqui. Bras. Odontopediatria Clín Integr. 2012;12(1):143-48.

10. Waskievicz AL, Baldissareli F, Vanni JR, Hartmann MSM, Fornari VJ. Avaliação da dor pós-operatória em dentes tratados endodonticamente.J Oral Invest.2013;2(1):43-8.

11. Alí A, Olivieri JG, Duran-Sindreu F, Abella $F$, Roig $M$, García-Font $M$. Influence of preoperative pain intensity on postoperative pain after root canal treatment: A prospective clinical study. J Dent. 2015;45:39-42.

12. Oliveira RKF, Rocha MP. Sintomatologia dolorosa após tratamento endodôntico: Revisão da literatura. Id on Line Ver Mult Psic. 2018;12 (42):696-703.

13. Su Y, Wang C, Ye L. Healing rate and postobturation pain of single-versus multiple-visit endodontic treatment for infected root canals: a systematic review. J Endod. 2011;37(2):125-32.

14. Singh S, Garg A. Incidence of post-operative pain after single visit and multiple visit root canal treatment: A randomized controlled trial. J Conser Dent. 2012;15(4):323-27.

15. Endo MS, Santos ACL, Pavan AJ, Queiroz AF, Pavan NNO. Endodontia em sessão única ou múltipla: revisão da literatura. RFO UPF. 2015; 20(3):408-13.

16. Rosa JCG. Tratamento Endodôntico em sessão única e dor pós-operatória [monografia]. Pouso Alegre: Instituto Nacional de Ensino Superior e Pós-Graduação Padre Gervásio - INAPÓS; 2015.

17. Manfredi M, Figini L, Gagliani M, Lodi G. Single versus multiple visits for endodontic treatment of permanent teeth. COCHRANE DB Syst Rev. 2016;(12).

18. Ribeiro SLBP, Maldonado VM, Nascimento VR, Tomazinho LF. Endodontic treatment: factors related to postoperative pain and the success of therapy. Braz J Surg Clin Res. 2016;16(2):9-13.

19. El Mubarak $\mathrm{AH}$, Abu-bakr NH, Ibrahim YE. Postoperative pain in multiple-visit and singlevisit root canal treatment. J Endod. 2010; 36(1):36-9.

20. Farzana $F$, Hossain SMI, Islam SMN, Rahman MA. Postoperative pain following multi-visit root canal treatment of teeth with vital and non-vital pulps. J Armed Forces Med Coll. 2010;6(2): 28-31.

21. Gurgel-Filho ED, Castelo-Branco YN, ManigliaFerreira C, de Souza-Filho FJ, Coutinho-Filho T. Avaliação in vivo da dor pós-operatória em dentes vitais após o alargamento do forame apical. RFO UPF. 2010;15 (2):145-49.

22. Kirchhoff AL, Fariniuk LF, Mello I. Apical extrusion of debris in flat-oval root canals after using different instrumentation systems. J Endod. 2015; 41(2):237-41.

23. Neelakantan $P$, Sharma S. Pain after singlevisit root canal treatment with two single-file systems based on different kinematics-a prospective randomized multicenter clinical study. Clin Oral Investig. 2015;19(9):2211-217.

24. Cruz-Júnior JA, Coelho MS, Kato AS, Vivacqua-Gomes N, Fontana CE, Rocha DGP et al. The effect of foraminal enlargement of necrotic teeth with the reciproc system on postoperative pain: a prospective and randomized clinical trial. J Endod. 2016;42 (1):8-11.

25. Saini HR, Sangwan P, Sangwan A. Pain following foraminal enlargement in mandibular molars with necrosis and apical periodontitis: a randomized controlled trial. Int Endod J. 2016; 49(12):1116-123.

26. Cicek E, Kocak MM, Kocak S, Saglam BC, Türker SA. Postoperative pain intensity after using different instrumentation techniques: a randomized clinical study. J Appl Oral Sci. 2017;25(1):20-6.

27. Hou XM, Su Z, Hou BX. Post endodontic pain 
following single-visit root canal preparation with rotary vs reciprocating instruments: a metaanalysis of randomized clinical trials. BMC Oral Health. 2017;17(1):86-93.

28. Souza ACA, Martins CM, Batista VES, Andrada AC, Mori GG, Gomes Filho JE. Influência da cinemática automatizada (sistema rotatório e reciprocante) na dor pós-operatória do tratamento endodôntico: revisão sistemática e meta-análise de estudos clínicos randomizados. Arch Health Invest. 2017:6 (Special Issue 6):28.

29. Ribeiro APD, Malnati PDS, Costa-Júnior ED. Limpeza do forame e extrusão apical de raspas de dentina em dentes unirradiculares submetidos ao procedimento de patência apical. Pesqui. Bras. Odontopediatria Clín. Integr. 2010;10(1):55-60.

30. Tuncer AK, Gerek M. Effect of working length measurement by electronic apex locator or digital radiography on postoperative pain: a randomized clinical trial. J Endod. 2014;40 (1):38-41.

31. Arora M, Sangwan P, Tewari S, Duhan J. Effect of maintaining apical patency on endodontic pain in posterior teeth with pulp necrosis and apical periodontitis: a randomized controlled trial. Int Endod J. 2016;49(4):317-24.

32. Pauletto G, Bello MDC. O impacto da patência apical para 0 sucesso do tratamento endodôntico: uma revisão de literatura. RFO UPF. 2018;23 (3):382-88.

33. Asgary S, Eghbal MJ. The effect of pulpotomy using a calcium-enriched mixture cement versus one-visit root canal therapy on postoperative pain relief in irreversible pulpitis: a randomized clinical trial. Odontology. 2010; 98(2):126-33.

34. Bashetty K, Hedge J. Comparison of $2 \%$ chlorhexidine and $5.25 \%$ sodium hypochlorite irrigating solutions on postoperative pain: a randomized clinical trial. Indian J Dent Res. 2010;21(4):523-27.

35. Almeida G, Marques E, Martins AS, Silveira Bueno CE, Nowakowski A, Cunha RS. Influence of irrigating solution on postoperative pain following single-visit endodontic treatment: randomized clinical trial. J Can Dent Assoc. 2012;78:c84.

36. Singh RD, Khatter R, Bal RK, Bal CS. Intracanal medications versus placebo in reducing postoperative endodontic pain-a double-blind randomized clinical trial. Braz Dent J. 2013;24(1):25-9.

37. Anjaneyulu K, Nivedhitha MS. Influence of calcium hydroxide on the post-treatment pain in endodontics: A systematic review. J Conserv Dent. 2014;17(3):200-7.
38. Vieyra JP, Enriquez FJJ, Acosta FO, Guardado JA. Reduction of postendodontic pain after one-visit root canal treatment using three irrigating regimens with different temperature. Niger J Clin Pract. 2019; 22(1):34-40.

39. Gambarini G, Testarelli L, De-Luca M, Milana $\mathrm{V}$, Plotino $\mathrm{G}$, Maria-Grande $\mathrm{N}$, et al. The influence of three different instrumentation techniques on the incidence of postoperative pain after endodontic treatment. Ann Stomatol. 2013;4(1):152-55.

40. Kherlakian D, Cunha RS, Ehrhardt IC, Zuolo ML, Kishen A, Silveira-Bueno CE. Comparison of the incidence of postoperative pain after using 2 reciprocating systems and a continuous rotary system: a prospective randomized clinical trial. J Endod. 2016;42(2):171-76.

41. Relvas JBF, Bastos MMB, Marques AAF, Garrido ADB, Sponchiado-Jr EC. Assessment of postoperative pain after reciprocating or rotatory $\mathrm{NiTi}$ instrumentation of root canals: a randomized, controlled clinical trial. Clin Oral Investig. 2016;20(8):1987-993.

42. Ensinas $\mathrm{P}$, Caba-Cabrera R, Martel M, Cornejo $\mathrm{N}$, Ramos L, Rionda $\mathrm{R}$ et al. Estudio clínico prospectivo del dolor posoperatorio en pacientes, asociado al uso de la lima de pasaje. Rev Asoc Odontol Argent. 2015;103 (2):81-5.

43. Bourreau MLS, Soares AJ, Souza-Filho FJ. Evaluation of postoperative pain after endodontic treatment with foraminal enlargement and obturation using two auxiliary chemical protocols. Rev Odontol UNESP. 2015; 44(3):157-62.

44. Silva EJNLD, Monteiro MR, Belladonna FG, Almeida JF, De-Deus G, Neves ADA. Postoperative pain after foraminal instrumentation with a reciprocating system and different irrigating solutions. Braz Dent J. 2015; 26(3):216-21.

\section{CONFLITO DE INTERESSES}

Os autores declaram não haver conflitos de interesse

\section{AUTOR PARA CORRESPONDÊNCIA}

José Henrique de Araújo Cruz
Rua Paulo Diogenes no 57, Centro
59990000 Rafael Fernandes - RN, Brasil
Telefone: (83) 99625-0125
E-mail: henrique_araujo1992@hotmail.com

Submetido em 09/04/2020 Aceito em 23/10/2020 